\title{
A pedagogical approach to Magnus expansion
}

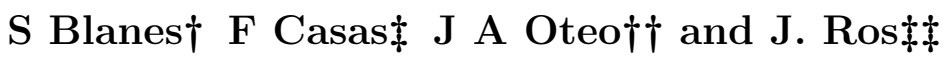

$\dagger$ Instituto de Matemática Multidisciplinar, Universidad Politécnica de Valencia, E-46022 Valencia, Spain

‡ Departament de Matemàtiques, Universitat Jaume I, E-12071 Castellón, Spain

†† Departament de Física Teòrica, Universitat de València, 46100-Burjassot, València, Spain

括 Departament de Física Teòrica and Instituto de Física Corpuscular(IFIC), Universitat de València, 46100-Burjassot, València, Spain

E-mail: serblaza@imm.upv.es, casas@mat.uji.es, oteo@uv.es, rosjouv.es

\begin{abstract}
Time-dependent perturbation theory as a tool to compute approximate solutions of the Schrödinger equation does not preserve unitarity. Here we present, in a simple way, how the Magnus expansion (also known as exponential perturbation theory) provides such unitary approximate solutions. The purpose is to illustrate the importance and consequences of such a property. We suggest that Magnus expansion may be introduced to students in advanced courses of Quantum Mechanics.
\end{abstract}

PACS numbers: 02.30.Mv, 03.65.Nk, 03.65.Db, 03.65.Fd, 02.30.Hq

AMS classification scheme numbers: 81Q15, 81V99, 41A58

Submitted to: European Journal of Physics 


\section{Motivation}

In Physics courses the key role of a few first principles in the formulation of the basic laws is emphasized. Students are then, from the very beginning, exposed to the idea that the fundamental equations have to strictly obey those requirements. Soon, however, when they come to applications they face the fact that exact solution of these equations is a rare possibility and consequently approximations are necessary.

A question then naturally arise: do these approximate treatments still satisfy the basic principles requisites? For some of the more conventional approaches the answer is often in the negative. It is then interesting to discuss to what extent can one devise approximate algorithms which are still respectful of the general principles.

In this paper we consider this issue taking as an example time evolution in Quantum Mechanics. Our presentation will be addressed to Physics or Engineering students with some general ideas of Quantum Mechanics and with elementary knowledge of differential equations and matrix calculus.

During the time evolution of a quantum system the total probability of the possible outcomes of any measurement on it is conserved. Or, in mathematical terms, the evolution operator $U\left(t, t_{0}\right)$ transforming state at time $t_{0}$ into state at time $t$ is unitary. However, when the corresponding time-dependent Schrödinger equation is solved by the most familiar approximation schemes the unitarity condition is lost.

Here we present the Magnus expansion (ME) [1]: a systematic way to build approximations to the time-dependent Schrödinger equation in such a way that, in any order, the evolution operator is unitary. It has a long history of more than 50 years and has been, and still is, widely used. However it seems to have found no place in the graduate curriculum, far less at the undergraduate level, as the lack of elementary expositions indicates. An interesting exception is reference [2], which formally unifies different unitary expansions but without illustrative examples. Our exposition will be kept within pedagogical limits and, consequently, questions of mathematical rigor and further developments will intentionally be left out. The interested reader is referred to [3] for a more detailed recent review where also a more complete list of references may be found.

The paper is organized as follows: in section 2 the conventional textbook treatment of the time evolution operator is roughly summarized pointing out its conflict with unitarity requirement. In section 3 the Magnus expansion is deduced in an as direct as possible way. The first three terms are explicitly given and their relation with the conventional time-dependent perturbation theory established. Section 4 includes two examples to illustrate how the algorithm works. The Conclusions section collects some final comments. 


\section{Customary time-dependent perturbation theory}

Let us consider a quantum system with a time-dependent Hamiltonian

$$
\mathcal{H}(t)=\mathcal{H}_{0}+\lambda \mathcal{H}(t)
$$

where $\mathcal{H}_{0}$ is time-independent. $\lambda$ is a bookkeeping parameter which at the end can be taken $\lambda=1$.

As one can find in most textbooks of Quantum Mechanics, in order to study the time evolution of our system the first step consists in performing what is called a change of picture, disposing of the (assumed known) action of $\mathcal{H}_{0}$.

In section 4 that transformation process will be illustrated. For the time being let us denote the transformed Hamiltonian by $\lambda H(t)$ and study the evolution associated with it.

To this aim one defines the time-evolution operator $U\left(t, t_{0}\right)$ which evolves the wave function $\Psi$ between times $t_{0}$ and $t$ as $\Psi(t)=U\left(t, t_{0}\right) \Psi\left(t_{0}\right)$. It is a unitary operator: $U\left(t, t_{0}\right) U^{\dagger}\left(t, t_{0}\right)=U^{\dagger}\left(t, t_{0}\right) U\left(t, t_{0}\right)=I$. This unitary character of $U$ keeps constant the norm of the wave function and ensures probability conservation. The Schrödinger equation for $U$ reads

$$
i \hbar \frac{\partial}{\partial t} U\left(t, t_{0}\right)=\lambda H(t) U\left(t, t_{0}\right)
$$

with initial condition $U\left(t_{0}, t_{0}\right)=I$.

From the mathematical point of view the basic objects we deal with $(H, U)$ and their relatives are operators in the Hilbert space of quantum states. However, for the sake of simplicity, we suggest to the inexperienced reader to think on them simply as (finite) dimensional matrices, and as such will be referred to indistinctively.

Equation (2) is easily solved by iteration. This procedure is equivalent to time-dependent perturbation theory (TDPT), and gives the expansion

$$
U\left(t, t_{0}\right)=I+\sum_{n=1}^{\infty} \lambda^{n} P_{n}\left(t, t_{0}\right),
$$

where $P_{n}\left(t_{0}, t_{0}\right)=0$. Substituting in (2) and comparing terms of the same order in $\lambda$ we find

$i \hbar \frac{\partial}{\partial t} P_{1}\left(t, t_{0}\right)=H(t), \quad i \hbar \frac{\partial}{\partial t} P_{n}\left(t, t_{0}\right)=H(t) P_{n-1}\left(t, t_{0}\right) \quad(n>1)$,

whence

$$
P_{n} \equiv P_{n}\left(t, t_{0}\right)=(-i / \hbar)^{n} \int_{t_{0}}^{t} \mathrm{~d} t_{1} \cdots \int_{t_{0}}^{t_{n-1}} \mathrm{~d} t_{n} H_{1} \cdots H_{n},
$$

where we have shortened $H_{i} \equiv H\left(t_{i}\right)$.

If the Hamiltonian does not depend on time, then (5) yields

$$
P_{n}=\frac{1}{n !}\left(-i\left(t-t_{0}\right) H / \hbar\right)^{n}
$$


and the sum in (3) gives the familiar expression

$$
U\left(t, t_{0}\right)=\exp \left(-i\left(t-t_{0}\right) \lambda H / \hbar\right),
$$

which also follows by direct integration of (2) in this case. In this form the result explicitly shows the unitary character of $U\left(t, t_{0}\right)$ : any matrix of the form $\exp (i A)$ with $A$ Hermitian is always unitary. Notice that the previous equation may be only deceptively simple: matrix exponentials are not always easy to calculate! As we will see this last comment will also apply to the Magnus expansion.

When the Hamiltonian is time-dependent, however, no such a simple form is obtained. Of course the sum of the whole series (3) provides a unitary operator, but in practice one has to truncate the series and the result is not unitary any longer. It may be instructive to compare the situation with a very familiar one: the sine function is certainly periodic, but any truncation whatsoever of its series expansion ceases to share exactly that property.

The non-unitary character of finite order TDPT does not mean that this is a useless scheme. On the contrary, the theory has been successfully in use ever since the beginning of Quantum Mechanics. Hence the student should not loose interest in grasping the TDPT techniques. Keep in mind that every approximate method of solution has its own limitations and the one we will explain in the next section does not escape the rule.

Here a comment may be in order: equation (7) may be trivially written as

$$
U\left(t, t_{0}\right)=\exp \left(-\frac{i}{\hbar} \lambda \int_{t_{0}}^{t} H\left(t^{\prime}\right) \mathrm{d} t^{\prime}\right) .
$$

In the time-dependent case neither equations (7) nor (8) are valid. But, as can be seen in some advanced textbooks on Quantum Mechanics, it is customary to introduce the so-called time-ordering or Dyson operator $\mathcal{T}$ defined as

$$
\mathcal{T}\left(H\left(t_{1}\right) H\left(t_{2}\right)\right)= \begin{cases}H\left(t_{1}\right) H\left(t_{2}\right) & t_{1}>t_{2}, \\ \left.H\left(t_{2}\right) H\left(t_{1}\right)\right) & t_{2}>t_{1}\end{cases}
$$

and then $U\left(t, t_{0}\right)$ can symbolically be written like

$$
U\left(t, t_{0}\right)=\mathcal{T}\left(\exp \left(-\frac{i}{\hbar} \lambda \int_{t_{0}}^{t} H\left(t^{\prime}\right) \mathrm{d} t^{\prime}\right)\right)
$$

which, in spite of its appearance does not correspond to a true exponential representation, at variance with (8).

\section{Magnus expansion}

The so-called Magnus expansion (ME) [1], also referred to as exponential perturbation theory in the literature, starts by assuming that a true exponential solution of (2) exists:

$$
U\left(t, t_{0}\right)=\exp \left(\Omega\left(t, t_{0}\right)\right)=I+\sum_{n=1}^{\infty} \frac{1}{n !} \Omega^{n}, \quad \Omega\left(t_{0}, t_{0}\right)=0 .
$$


and proceeds to find and solve by series expansion an equation for $\Omega\left(t, t_{0}\right)$.

For ease of reading, from now on we change our notations a little bit. In the previous section we have explicitly written the $i$ and $\hbar$ factors as is usually done. However, it will prove convenient to stick those factors to the Hamiltonian defining

$$
\tilde{H}(t) \equiv-i H(t) / \hbar
$$

and the Schrödinger equations for $U\left(t, t_{0}\right)$ looks now:

$$
\frac{\partial}{\partial t} U\left(t, t_{0}\right)=\lambda \tilde{H}(t) U\left(t, t_{0}\right)
$$

with initial condition $U\left(t_{0}, t_{0}\right)=I$.

Observe that the Hermitian character of $H$ makes $\tilde{H}$ antihermitian, which simply means $\tilde{H}^{\dagger}=-\tilde{H}$. The previously quoted result about the exponential representation of unitary matrices can be now rephrased as saying that any unitary matrix is the exponential of an antihermitian matrix. So, we get our first condition on $\Omega\left(t, t_{0}\right)$ : it has to be antihermitian in order to ensure the unitary character of $U\left(t, t_{0}\right)$.

For future reference, notice that if $A$ and $B$ are two antihermitian operators then their commutator, $[A, B] \equiv A B-B A$, is also antihermitian. This property will be instrumental in proving the unitary character of the Magnus scheme.

Notice that by changing our unknown from $U$ to $\Omega$ we look for the logarithm of the time-evolution operator. In any case, if the Hamiltonian is time-independent then, according to the preceding section $\Omega\left(t, t_{0}\right)=\left(t-t_{0}\right) \tilde{H}$.

To see the origin of the difficulties when the Hamiltonian is time-dependent let us consider the differential equation (13) but taking $U$ and $\tilde{H}$ as ordinary scalar functions instead of matrices. From elementary Calculus we know that the solution is

$$
U\left(t, t_{0}\right)=\exp \left(\lambda \int_{t_{0}}^{t} \tilde{H}\left(t^{\prime}\right) \mathrm{d} t^{\prime}\right) .
$$

as it is immediately verified by direct ordinary differentiation.

The key point is that if, instead, we deal with matrices the familiar rule

$$
\frac{\mathrm{d}}{\mathrm{d} t} \exp (A(t))=A^{\prime}(t) \exp (A(t))=\exp (A(t)) A^{\prime}(t)
$$

is not generally valid any more because matrices $A(t)$ and $A^{\prime}(t)$ could be noncommuting. The exponential in equation (14) is not a solution of (13) unless $\left[\int_{t_{0}}^{t} \tilde{H}\left(t^{\prime}\right) \mathrm{d} t^{\prime}, H(t)\right]=0$, or equivalently $\left[H\left(t_{1}\right), H\left(t_{2}\right)\right]=0$, for arbitrary $t_{1}, t_{2}$. Obviously, this is certainly the case if $H$ is time-independent. Otherwise, the search of $\Omega$ becomes much more difficult in general.

The question now is: If $U$ obeys (13) then what is the evolution equation for $\Omega$ ? Inasmuch as we are not allowed to use the familiar differentiation rules we have to follow a different route to proceed. Here two important results come to our rescue. 
The first one is the, intuitively clear, so-called group property of the evolution operator:

$$
U\left(t_{2}, t_{0}\right)=U\left(t_{2}, t_{1}\right) U\left(t_{1}, t_{0}\right) .
$$

The second one is related to a famous classical result in matrix algebra known as the Baker-Campbell-Haussdorf $(\mathrm{BCH})$ formula [4] for the product of two exponentials. It states that for any two, in general noncommuting, operators $X$ and $Y$

$\exp X \exp Y=\exp \left(X+Y+\frac{1}{2}[X, Y]+\frac{1}{12}[X,[X, Y]]+\frac{1}{12}[Y,[Y, X]]+\ldots\right)$

The exponent in this equation is an infinite series. Its terms are nested commutators of increasing order.

The simplicity in (17) is deceptive because higher order terms become quickly very much involved. The explicit form of the series is not known, although there exist algorithms to compute it to finite order. As a measure of its complexity, we mention that the number of terms in order $m$ is $\mathcal{O}\left(2^{m} / m\right)$ and the CPU time increases consequently.

In spite of this awful complexity, surprisingly, a compact formula exists [5, 6] that gives the piece of the $\mathrm{BCH}$ series to all orders in one operator, say $Y$, and to first order in $X$, namely

$$
\operatorname{expX} \exp Y=\exp \left(Y+\sum_{k=0}^{\infty} \frac{B_{k}}{k !}\left\{Y^{k}, X\right\}+\mathcal{O}\left(X^{2}\right)\right)
$$

where $\left\{Y^{k}, X\right\}=[Y,[Y, \ldots], X]$ stands for a $k$-times nested commutator. By definition $\left\{Y^{0}, X\right\}=X$. $B_{k}$ are Bernoulli numbers [7].

Now, to derive the equation satisfied by $\Omega$ we consider a narrow time interval $\delta t$ after time $t$, which at the end we want to let $\delta t \rightarrow 0$, and use the group property (16) with $t_{2}=t+\delta t, t_{1}=t$ to write $U\left(t+\delta t, t_{0}\right)=U(t+\delta t, t) U\left(t, t_{0}\right)$. In exponential form it reads

$$
\exp \left(\Omega\left(t+\delta t, t_{0}\right)\right)=\exp (\Omega(t+\delta t, t)) \exp \left(\Omega\left(t, t_{0}\right)\right) .
$$

The crucial point now is that, during the interval $(t, t+\delta t)$ the Hamiltonian can be assumed to take the constant value $\tilde{H}(t)$. The Schrödinger equation can be solved in the exponential form $\exp (\Omega(t+\delta t, t)) \simeq \exp (\lambda \tilde{H}(t) \delta t)$ and so

$$
\exp \left(\Omega\left(t+\delta t, t_{0}\right)\right) \simeq \exp (\lambda \tilde{H}(t) \delta t) \exp \left(\Omega\left(t, t_{0}\right)\right) .
$$

Introducing (18) into (20) and keeping just the first order in $\delta t$ we get

$$
\Omega\left(t+\delta t, t_{0}\right)=\Omega\left(t, t_{0}\right)+\delta t \lambda \sum_{k=0}^{\infty} \frac{B_{k}}{k !}\left\{\Omega^{k}\left(t, t_{0}\right), \tilde{H}\right\}+\mathcal{O}\left(\delta t^{2}\right)
$$

So, in the limit $\delta t \rightarrow 0$ we get

$$
\frac{\partial}{\partial t} \Omega\left(t, t_{0}\right)=\lambda \sum_{k=0}^{\infty} \frac{B_{k}}{k !}\left\{\Omega^{k}\left(t, t_{0}\right), \tilde{H}(t)\right\}, \quad \Omega\left(t_{0}, t_{0}\right)=0,
$$


which is a highly nonlinear differential equation for $\Omega$, in contrast with the linear differential equation obeyed by $U$. So far, finding approximate solutions for the logarithm of the time-evolution operator may seem a more difficult task than finding $U$ itself.

The gain with the exponential representation of the time-evolution operator emerges when $\Omega$ is expanded in power series in $\lambda$ and introduced in (22). It is then natural to expand $\Omega=\sum_{k=1}^{\infty} \lambda^{k} \Omega_{k}$, which we call the Magnus series. Equating terms of same order in $\lambda$, we break the previous differential equation (22) into an infinite set of trivially integrable differential equations for each $\Omega_{k}$. After some algebra the first three terms read

$\Omega_{1}\left(t, t_{0}\right)=\int_{t_{0}}^{t} \tilde{H}\left(t_{1}\right) \mathrm{d} t_{1}$,

$\Omega_{2}\left(t, t_{0}\right)=\frac{1}{2} \int_{t_{0}}^{t} \mathrm{~d} t_{1} \int_{t_{0}}^{t_{1}} \mathrm{~d} t_{2}\left[\tilde{H}\left(t_{1}\right), \tilde{H}\left(t_{2}\right)\right]$

$\Omega_{3}\left(t, t_{0}\right)=\frac{1}{6} \int_{t_{0}}^{t} \mathrm{~d} t_{1} \int_{t_{0}}^{t_{1}} \mathrm{~d} t_{2} \int_{t_{0}}^{t_{2}} \mathrm{~d} t_{3}\left(\left[\tilde{H}_{1},\left[\tilde{H}_{2}, \tilde{H}_{3}\right]\right]+\left[\tilde{H}_{3},\left[\tilde{H}_{2}, \tilde{H}_{1}\right]\right]\right)$,

where again we have abbreviated $\tilde{H}\left(t_{i}\right) \equiv \tilde{H}_{i}$. Observe that $\Omega_{1}$ originates, after exponentiation, the trial (14). But now it is only part of the solution. Interestingly, $\Omega_{k}$, with $k>1$, are the successive corrections originated by the nonvanishing commutators, necessary to have an exponential solution for $U\left(t, t_{0}\right)$.

As a rule of thumb we can say that to obtain $\Omega_{k}$ all one has to do is commute and integrate. The important point is that, by so doing to any order, the truncated sum of series for $\Omega$ is always antihermitian, because of our previous comment for the commutator of antihermitian operators. Consequently its exponential furnishes an unitary approximation for $U\left(t, t_{0}\right)$. That is precisely what we were looking for.

From a practical point of view two comments are in order. First, equations (23) give explicitly the first terms of the Magnus series. It can also be proved that all terms in the series can be obtained recursively. Second, as was already mentioned, once an expression for $\Omega$ is obtained one still has to calculate its exponential; and this may not be an easy task.

The connection between perturbative and Magnus series may be readily established from the expansions

$$
U=I+\lambda P_{1}+\lambda^{2} P_{2}+\cdots=\exp \left(\lambda \Omega_{1}+\lambda^{2} \Omega_{2}+\cdots\right) .
$$

Thus, we have

Conversely

$$
\begin{aligned}
& P_{1}=\Omega_{1}, \\
& P_{2}=\Omega_{2}+\frac{1}{2 !} \Omega_{1}^{2}, \\
& P_{3}=\Omega_{3}+\frac{1}{2 !}\left(\Omega_{1} \Omega_{2}+\Omega_{2} \Omega_{1}\right)+\frac{1}{3 !} \Omega_{1}^{3} .
\end{aligned}
$$

$$
\Omega_{2}=P_{2}-\frac{1}{2 !} P_{1}^{2}
$$




$$
\Omega_{3}=P_{3}-\frac{1}{2}\left(P_{1} P_{2}+P_{2} P_{1}\right)+\frac{1}{3} P_{1}^{3} .
$$

\section{Two illustrative Examples}

For the sake of illustration of ME and comparison with TDPT this section presents a simplified version of application examples given in $[3,8]$. In the two examples we treat, the use of approximation methods is, in fact, purely illustrative because for both the exact solution is known. But the comparison between approximate and exact results buttress the importance of calculating with unitary approximations. We deal with the first and second approximants in ME and TDPT for the harmonic oscillator and two-level spin problems.

The Hamiltonian in these examples has the structure $H(t)=H_{0}+V(t)$, where $H_{0}$ stands for the solvable piece of the problem and is independent of time. $V(t)$ is a small perturbation. Solvable means that the exact time-displacement operator associated to $H_{0}$ is known and reads $U_{0}(t)=\exp \left[\left(t-t_{0}\right) \tilde{H}_{0}\right]$. Here the bookkeeping parameter from the previous sections has been set to $\lambda=$ 1. This splitting of the Hamiltonian will allow us to focus the perturbative approximations just on the piece $V(t)$.

As we mentioned in section 2 to proceed further on we have to express the Hamiltonian in the so-called Interaction Picture. Mathematically, it means that the solvable piece $H_{0}$ is exactly integrated via the factorization $U=U_{0} U_{I}$, where the time-evolution operator in the Interaction Picture obeys the Schrödinger equation

$$
\frac{\partial}{\partial t} U_{I}\left(t, t_{0}\right)=\tilde{H}_{I}(t) U_{I}\left(t, t_{0}\right), \quad U_{I}\left(t_{0}, t_{0}\right)=I,
$$

with

$$
\tilde{H}_{I}(t) \equiv e^{\left(t-t_{0}\right) \tilde{H}_{0}} \tilde{V}(t) e^{-\left(t-t_{0}\right) \tilde{H}_{0}} .
$$

As a benchmark of our computations we will study the transition probability between two eigenstates $|a\rangle$ and $|b\rangle$ of $H_{0}$

$$
\mathcal{P}(a \rightarrow b)=\left|\left\langle b\left|U\left(t, t_{0}\right)\right| a\right\rangle\right|^{2}=\left|\left\langle b\left|U_{I}\left(t, t_{0}\right)\right| a\right\rangle\right|^{2} .
$$

\subsection{Linearly forced harmonic oscillator}

The linearly forced harmonic oscillator is a soluble problem in Quantum Physics that has received a great deal of attention along the years. The Hamiltonian reads: $H=H_{0}+V(t)$, where $H_{0}$ stands for the unperturbed Hamiltonian

$$
H_{0}=a_{+} a_{-}+1 / 2,
$$

and $V(t)$ is the perturbation

$$
V(t)=f(t)\left(a_{+}+a_{-}\right),
$$

written in terms of raising and lowering (i.e. creation and annihilation) operators with commutation relation $\left[a_{-}, a_{+}\right]=1$. We have used units in which mass, 
frequency, and Planck constant are unity $(m=\omega=\hbar=1) . f(t)$ is an small arbitrary scalar function of $t$.

A bit of algebra yields the Hamiltonian in the Interaction Picture: $H_{I}(t)=$ $f(t)\left(e^{i t} a_{+}+e^{-i t} a_{-}\right)$. For future reference notice that this operator, when sandwiched between eigenstates $|m\rangle$ and $\left|m^{\prime}\right\rangle$ of $H_{0}$, can only connect states with $\left|m-m^{\prime}\right|=1$.

The second order in ME needs the commutator $\left[H_{I}\left(t_{1}\right), H_{I}\left(t_{2}\right)\right]=$ $2 i f\left(t_{1}\right) f\left(t_{2}\right) \sin \left(t_{2}-t_{1}\right)$. As this is a scalar quantity, higher order terms in $\mathrm{ME}$ vanish and the series terminates, eventually yielding the exact solution. Explicitly, introducing

$\alpha=\int_{t_{0}}^{t} f\left(t_{1}\right) e^{i t_{1}} \mathrm{~d} t_{1}, \quad \beta=\int_{t_{0}}^{t} \mathrm{~d} t_{1} \int_{t_{0}}^{t_{1}} f\left(t_{1}\right) f\left(t_{2}\right) \sin \left(t_{2}-t_{1}\right) \mathrm{d} t_{2}$,

we have

$$
\begin{aligned}
& \Omega_{1}^{I}\left(t, t_{0}\right)=-i\left(\alpha a_{+}+\alpha^{*} a_{-}\right) \\
& \Omega_{2}^{I}\left(t, t_{0}\right)=-i \beta \\
& \Omega^{I}\left(t, t_{0}\right)=\Omega_{1}^{I}+\Omega_{2}^{I}, \\
& U_{I}\left(t, t_{0}\right)=\exp \left(\Omega^{I}\left(t, t_{0}\right)\right)=\exp \left[-i\left(\alpha a_{+}+\alpha^{*} a_{-}\right)-i \beta\right],
\end{aligned}
$$

where the superscript $I$ indicates that the computation has been made in the Interaction Picture.

In the perturbative expansion, $P_{1}^{I}$ and $P_{2}^{I}$ can be directly obtained from (25). Observe that, contrary to what happens with ME, the series (3) does not terminate. It is clear that in second order TDPT

$$
U\left(t, t_{0}\right) \simeq U_{0}\left(t, t_{0}\right)\left(I+P_{1}^{I}\left(t, t_{0}\right)+P_{2}^{I}\left(t, t_{0}\right)\right)
$$

is not unitary.

This simple example reveals also an important physical difference between ME and TDPT: due to the fact that in any order of approximation ME furnishes an exponential representation of $U$ it compactly involves the action of an infinite number of creation-annihilation operators and thus connect any pair of eigenstates $|m\rangle$ and $\left|m^{\prime}\right\rangle$. On the contrary, TDPT in any finite order of approximation involves exclusively a finite number of $a_{ \pm}$operators and correspondingly the transition $|m\rangle \rightarrow\left|m^{\prime}\right\rangle$ has to satisfy strict selection rules in order to be allowed.

For the sake of illustration, let us suppose $f(t)$ even and $t=+\infty, t_{0}=-\infty$. Then $U(+\infty,-\infty)$ corresponds to the scattering $S$ matrix. The transition probability from the ground state $|0\rangle$ to the excited state $|m\rangle$ gives in ME the usual Poisson distribution $\mathcal{P}(0 \rightarrow m)=\alpha^{2 m} \exp \left(-\alpha^{2}\right) / m$ !. Whereas TDPT is, according with the comment above, absolutely unable to give such a result. Not to mention the further drawback that, due to the loss of unitarity, it may give $\mathcal{P}>1$, a fact that $\mathrm{ME}$ guarantees will never occur. 


\subsection{Two-level system}

Let us consider now a particle of spin $1 / 2$ evolving under the action of a magnetic field with components along the $x$ and $z$ directions. In terms of Pauli matrices (recall, $\left[\sigma_{x}, \sigma_{y}\right]=2 i \sigma_{z}$ ) the Hamiltonian reads

$$
H(t)=\frac{1}{2} \hbar \omega \sigma_{z}+f(t) \sigma_{x}
$$

and we take $f(t)=0$ if $t<0$ or $t>T$; and $f(t)=V_{0}$ if $0 \leq t \leq T$. Thus, $f(t)$ stands for a rectangular pulse.

In order to analyze the evolution induced by $H(t)$ we take $H_{0} \equiv \frac{1}{2} \hbar \omega \sigma_{z}$ as the unperturbed Hamiltonian. Its degenerate spin-up and spin-down eigenstates are denoted $| \pm\rangle$. Treating the remainder of (35) as a perturbation corresponds to $V_{0} \ll \hbar \omega$. Besides, $\omega T$ measures the ratio between the interaction time $T$ and the internal time of the system $1 / \omega$. Thus, $\omega T \ll 1$ and $\omega T \gg 1$ establish the sudden and adiabatic regimes of the perturbation $f(t)$, respectively.

If we take $t_{0}=0$ and $t>0$ the exact solution for $U(t, 0)$ is

$U_{e x}(t, 0)= \begin{cases}\exp \left(-i\left(\frac{\omega}{2} \sigma_{z}+\frac{V_{0}}{\hbar} \sigma_{x}\right) t\right), & 0 \leq t \leq T, \\ \exp \left(-i \omega(t-T) \sigma_{z} / 2\right) \exp \left(-i\left(\frac{\omega}{2} \sigma_{z}+\frac{V_{0}}{\hbar} \sigma_{x}\right) T\right), & t>T .\end{cases}$

The exponentials of Pauli matrices above may be evaluated by using the formula

$$
\exp (i \vec{a} \cdot \vec{\sigma})=I \cos (|\vec{a}|)+i \vec{a} \cdot \vec{\sigma} \sin (|\vec{a}|) /|\vec{a}|,
$$

valid for a linear combination of Pauli matrices that we express as the scalar product $\vec{a} \cdot \vec{\sigma}$ and where $I$ stands for the $2 \times 2$ unit matrix. Explicitly

$U_{e x}(t, 0)= \begin{cases}I \cos (\eta t / T)-\frac{i}{\eta}\left(\frac{\xi}{2} \sigma_{z}+\gamma \sigma_{x}\right) \sin (\eta t / T), & 0 \leq t \leq T, \\ b_{0} I-i \vec{b} \cdot \vec{\sigma}, & t>T,\end{cases}$

where

$$
\begin{aligned}
& b_{0}=\cos \tau \cos \eta-\frac{\xi}{2 \eta} \sin \tau \sin \eta, \\
& b_{1}=\frac{\gamma}{\eta} \cos \tau \sin \eta, \\
& b_{2}=\frac{\gamma}{\eta} \sin \tau \sin \eta, \\
& b_{3}=\frac{\xi}{2 \eta} \cos \tau \sin \eta+\sin \tau \cos \eta, \\
& \eta^{2}=\gamma^{2}+\xi^{2} / 4, \quad \gamma=V_{0} T / \hbar, \quad \xi=\omega T, \quad \tau=\omega(t-T) / 2 .
\end{aligned}
$$

The introduction of the dimensionless parameters $\gamma$ and $\xi$ will prove to be convenient later on. Notice that the first one stands for the pulse area and $\xi$ gives its duration, expressed in units of the internal time $1 / \omega$.

The time-evolution for $t>T$ simply mixes harmonically the eigenstates, as indicated by the $\tau$-dependent terms in (39). Hence, the interesting question is: Preparing the system at $t=0$ in an eigenstate, what is the relative proportion of each eigenstate just when the pulse vanishes, i.e., at $t=T$. Thus, as a test, we propose to compute the transition probability $\mathcal{P}(\xi)$ between the eigenstates $| \pm\rangle$ of $H_{0}$. 
To apply ME and TDPT to this problem we transform the Hamiltonian into the Interaction Picture defined by $H_{0}$ above

$$
\begin{aligned}
H_{I}(t) & =f(t) \exp \left(i H_{0} t / \hbar\right) \sigma_{x} \exp \left(-i H_{0} t / \hbar\right), \\
& =V_{0}\left(\sigma_{x} \cos (\omega t)-\sigma_{y} \sin (\omega t)\right), \quad 0 \leq t \leq T
\end{aligned}
$$

and $H_{I}=0$ elsewhere. The time-evolution operator gets factorized as $U(t, 0)=$ $\exp \left(-i \omega t \sigma_{z} / 2\right) U_{I}(t, 0)$. Due to the diagonal structure of the exponential, only $U_{I}$ is involved in the computation of $\mathcal{P}(t)$, namely $|\langle \pm|U| \mp\rangle|=\left|\left\langle \pm\left|U_{I}\right| \mp\right\rangle\right|$.

The first two orders in ME read

$$
\begin{aligned}
& \Omega_{1}(T, 0)=-i \frac{\gamma}{\xi}\left(\sigma_{x} \sin \xi+\sigma_{y}(1-\cos \xi)\right), \\
& \Omega_{2}(T, 0)=i\left(\frac{\gamma}{\xi}\right)^{2}(\xi-\sin \xi) \sigma_{z} .
\end{aligned}
$$

Using (37) we get the approximate transition probabilities

$\mathcal{P}_{M}^{(1)}(T)=\sin ^{2}\left(\frac{2 \gamma}{\xi} \sin \frac{\xi}{2}\right)$

$\mathcal{P}_{M}^{(2)}(T)=\left(\frac{2 \gamma}{\xi \mu} \sin \mu \sin \frac{\xi}{2}\right)^{2}, \quad \mu^{2}=4 \sin ^{2} \frac{\xi}{2}+\frac{\gamma^{2}}{\xi^{2}}(\xi-\sin \xi)^{2}$.

Time-dependent perturbation theory gives for the first and second approximation orders

$$
\mathcal{P}_{p t}^{(1)}(T)=\mathcal{P}_{p t}^{(2)}(T)=\left(\frac{2 \gamma}{\xi} \sin \frac{\xi}{2}\right)^{2} .
$$

Eventually, from (36) and (37) we get

$$
\mathcal{P}_{e x}(T)=\frac{4 \gamma^{2}}{4 \gamma^{2}+\xi^{2}} \sin ^{2} \sqrt{\gamma^{2}+\xi^{2} / 4}
$$

For the sake of illustration, in Figure 1 we plot the exact formula and the various approximations for $\mathcal{P}$ at the end of the pulse, as a function of $\xi$, with $\gamma=0.5$. For a fixed value of $\gamma, \xi \ll 1$ corresponds to the sudden limit whereas $\xi \gg 1$ is the adiabatic regime. Thus, the different systems compared in Figure 1 correspond to pulses of the same area, embracing the sudden and adiabatic regimes. Remind that $\xi$ is the dimensionless time expressed in units of internal time.

Notice that the limit of sudden pulse (i.e., $\xi \ll 1$ ) is very well described by ME, unlike TDPT. This is the general characteristic of ME approximations in the Interaction Picture. An intuitive explanation would be that the more sudden the perturbation the smaller the range of times where the commutator $\left[H_{I}\left(t_{1}\right), H_{I}\left(t_{2}\right)\right]$ does not vanish. Remind that this commutator is the origin of all the nuisances for the exponential representation of $U$. In the opposite limit, the adiabatic regime, the transition probability is very small. Both approaches seem to approximate well the exact result. This is, however, deceptive. In general, both ME and TDPT have to be preceded by a change of picture based on the instantaneous diagonal form of the full Hamiltonian if one wants to obtain meaningful transition 
probabilities. As a matter of fact, the preliminary transformation may be cast as an Adiabatic Picture.

In the example at hand, the second order correction of ME to the transition probability is very small whereas for TDPT it exactly vanishes. Moreover, main corrections come from odd approximation orders.

The inset if Figure 1 represents the relative error of the various approximants; i.e, the difference between the approximated and the exact probabilities, over the exact one. For ME the relative error fits a power-law (which corresponds to a straight line in log-log scales). For TDPT it is roughly constant in the interval plotted $(0<\xi<4)$. This behaviour persists with other values of $\gamma$.

Near the zero of $\mathcal{P}(\xi)$, around $\xi=6$, the relative error has no meaning. The interpretation of this zero is noteworthy. In that situation the system is unaffected by the perturbing pulse. The potential $V(t)$ presents a kind of transparency for this particular two-level configuration. Another consequence from Figure 1 is that the transitions between the two levels are most efficiently activated with sudden pulses.

\section{Conclusions}

In this paper we have shown how to build a perturbation expansion for the quantum time-evolution operator in such a way that truncation at any desired order of approximation does not spoil the desired unitary character of that operator. The proof presented is based on the group property of $U$ and focus all the mathematical stuff on a simplified form of the $\mathrm{BCH}$ formula.

We think that with the resources available to an average student of Quantum Mechanics the whole procedure can be followed and the topic could be introduced either directly in a general syllabus or as assigned work. It will help the student to see the noncommutativity of observables, one of the flagships of Quantum Mechanics, at work. In this instance it is not the case of two different observables like the familiar position and momentum, but the same observable at two different times.

As has been repeatedly emphasized, from the mathematical point of view, the complicated structure of the expansion will also give the opportunity for students to increase their skills in matrix calculus.

As announced in the first section our intention has only been to present the basic ideas of ME. Important questions, like convergence conditions, alternative approaches and generalizations have not been touched upon and the interested reader is referred to the literature. It is important to say that the Magnus proposal is by no means restricted the quantum Schrödinger equation but it applies directly to the linear differential operator initial value problem

$$
Y^{\prime}(t)=A(t) Y(t), \quad Y\left(t_{0}\right)=Y_{0},
$$

which pervades, in different guises, the entire field of Physics and probably the 
student has met the above equation in different contexts.

$\mathrm{ME}$ has found applications in a wide spectrum of fields from elementary particles, nuclear, atomic and molecular physics to classical dynamics, nuclear magnetic resonance or control theory. In recent years ME has found its way in the numerical treatment of differential equations, within the field of Geometric Integration, where it has been shown to provide effective algorithms which incorporates essential physical requirements $[3,9]$.

\section{Acknowledgments}

This work has been partially supported by contracts MCyT/FEDER, Spain (Grants No. MTM2007-61572 (SB, FC) and FIS2007-60133 (JAO, JR)) and $\mathrm{GV} / 2009 / 032$ (Generalitat Valenciana) (SB).

\section{References}

[1] Magnus W 1954 On the exponential solution of differential equations for a linear operator Commun. Pure Appl. Math. 7, 649-673.

[2] Fernández F M 2005 On the Magnus, Wilcox and Fer operator equations Eur. J. Phys. 26, 151-155.

[3] S. Blanes, F. Casas, J.A. Oteo, J. Ros 2009 The Magnus expansion and some of its applications, Physics Reports 470, 151-238.

[4] Wilcox R M 1967 Exponential operators and parameter differentiation in quantum physics J. Math. Phys. 8, 962-982.

[5] Klarsfeld S and Oteo J A 1989 The Baker-Campbell-Haussdorf formula and the convergence of the Magnus expansion J. Phys. A: Math. Gen. 22 , 4565-4572.

[6] Postnikov M M 1982 Lie Groups and Lie Algebras, URSS Publishers, p. 127

[7] Abramowitz M A and Stegun I A 1965 Handbook of Mathematical Functions (New York: Dover)

[8] Pechukas P and Light J C 1966 On the exponential form of time-displacement operators in Quantum Mechanics J. Chem. Phys. 44, 3897-3912.

[9] Iserles A, Munthe-Kaas H Z, Nørsett S P, and Zanna A 2000 Lie-group methods, Acta Numerica 9, 215-365. 


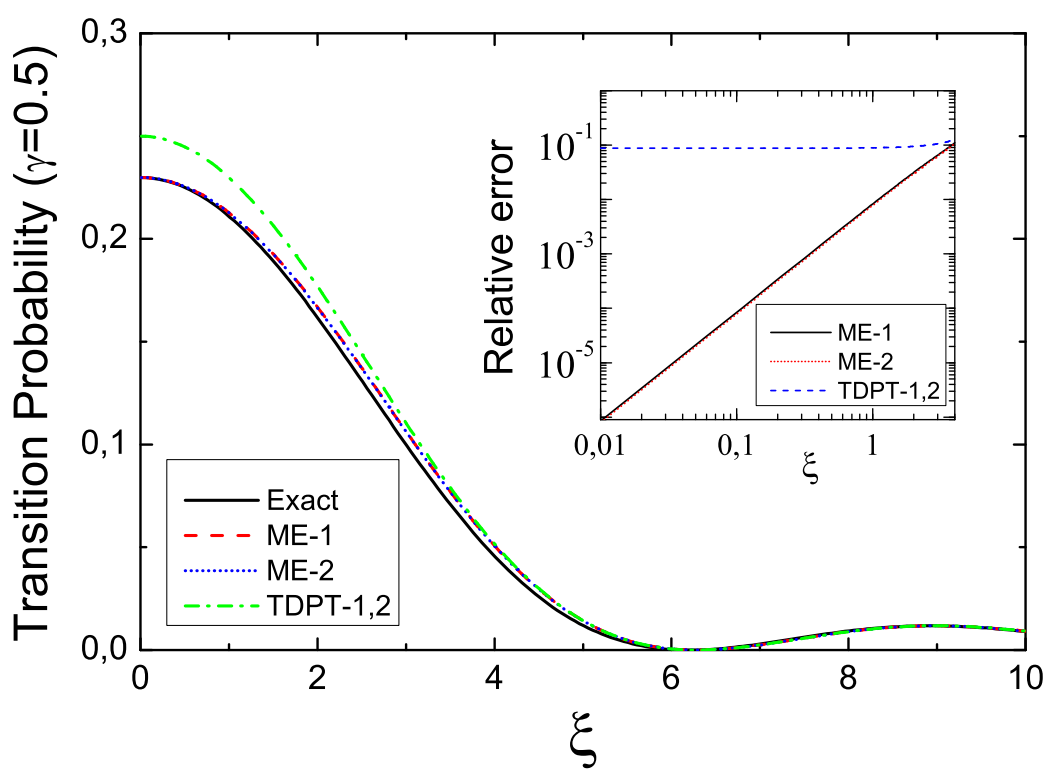

Figure 1. Two-level system: Transition probability as a function of the duration of the pulse $\xi$, for a pulse of area $\gamma=0.5$. First and second orders for Magnus approximants hardly differ. The inset stands for the relative error of the various approximations. 